\title{
How Sensor Graph Topology Affects Localization Accuracy
}

\author{
Deepti Kumar and Herbert G. Tanner
}

\begin{abstract}
We characterize the accuracy of a cooperative localization algorithm based on Kalman Filtering, as expressed by the trace of the covariance matrix, in terms of the algebraic graph theoretic properties of the sensing graph. In particular, we discover a weighted Laplacian in the expression that yields the constant, steady state value of the covariance matrix. We show how one can reduce the localization uncertainty by manipulating the eigenvalues of the weighted Laplacian. We thus provide insight to recent optimization results which indicate that increased connectivity implies higher accuracy. We offer an analysis method that could lead to more efficient ways of achieving the desired accuracy by controlling the sensing network.
\end{abstract}

\section{INTRODUCTION}

Every robot navigation and map building algorithm relies on accurate localization. Odometry is the simplest and most inexpensive technique for mobile robot localization, but unequal wheel diameters, uneven floor surface, wheel slippage, and low encoder resolution, contribute to errors that increase with time [1]. Position estimates are improved by means of relative position measurements to known landmarks [2], [3] made by exteroceptive sensors, such as laser range finders, sonar sensors, and infra-red sensors.

Several filters have been developed to fuse measurements obtained by the proprioceptive (internal measurement) and exteroceptive (external measurement) sensors like (Extended) Kalman filters [4], [5], particle filters [6], [7], grid based methods [8], [9] and expectation maximization algorithms [10], [11].

When landmarks are initially unavailable or sparsely populated, achieving the desired accuracy using these filters can be a challenge. In such cases, a robot team offers additional flexibility: robots within a team can improve their state estimates by using relative position measurements between themselves, in addition to measurements of known landmarks in the environment. In [12], the absence of available landmarks motivates the division of a group of robots into two teams. At a given instance of time, one of the teams acted as a landmark set for the other team. Over time, the roles can be reversed. If one can afford having one robot with absolute position information, then the combined (linearized) system becomes observable [5].

Not surprisingly, the larger the number of measurements, the better. However, sensor data availability always comes at a cost; computation requirements increase, and constraints on the spatial distribution of robots multiply. A question that arises is: how can we obtain a sufficiently good level of accuracy, with the smallest number of relative measurements?

Deepti Kumar and Herbert Tanner are with the Department of Mechanical Engineering at the University of New Mexico, Albuquerque, NM 87131.
The starting point in our approach to this question is [4], where authors compute upper bounds for the covariance matrix of position estimates obtained via the extended Kalman filter. In [13], they show that the trace is an non-convex objective function of the sensing graph topology, and they minimize it using a genetic algorithm. In this paper, we try to shed more light into the relation between the trace and the graph topology, by relating the two through the sensor graph weighted Laplacian. Although, as correctly observed in [13], the addition of edges in the sensing graph leads to improved localization accuracy, the claim made here is that some links are better than others. We do not offer a constructive way of designing the sparser sensing graph to fit given accuracy specifications, but this paper contributes by exposing the dependence of the trace to the weighted Laplacian eigenvalues. Hopefully, this link will enable us to invent efficient inter-robot sensing network architectures, drawing from the arsenal of algebraic graph theory results.

Section II formalizes our objective in this paper and states any additional assumptions. Section III reviews briefly how the closed form for the steady state value of the position estimates covariance matrix was derived in [4], for the particular case where one landmark's location is known accurately. We interpret a special case of [4] from an algebraic graph theoretic perspective in section IV, while section $\mathrm{V}$ gives proofs for interlacing theorems on weighted graphs.

\section{PROBLEM DESCRIPTION}

Consider a group of $M$ mobile robots capable of obtaining odometry data as well as making relative measurements with respect to each other. We assume the existence of at least one perfectly known landmark, which at least one of the robots can observe. Based on the absolute position information of this landmark, and relative position information between the robots in the group, all robots can localize themselves within certain error bounds, which depend on the statistics of their relative position measurements. The question in this paper is the following: How can one affect the accuracy of localization by changing the observation relations between the robots and the landmark?

\section{OUR STARTING POINT}

The material in this section is borrowed from [4], and the introductory discussion in this section attempts to set the stage and justify our interpretation of the results of [4], that follow in section IV. The mathematical development in this section is intentionally brief, since it is not new. We have kept notation as close to the original as possible, to facilitate 
the interested reader who is referred to [4] for details on the derivations.

Consider a team of $M$ mobile robots, moving in an area containing $N$ landmarks. The robots use proprioceptive measurements to propagate their own position estimates and obtain relative position estimates of neighboring robots using their exteroceptive sensors. An Extended Kalman Filter $(\mathrm{EKF})$ is used to filter out measurement noise and reduce the uncertainty associated with the interpretation of sensor signals. Each robot can obtain absolute orientation information.

Kalman filter estimation can be divided into two cycles: the position propagation cycle, in which the knowledge about position estimates is propagated to next time step; and the update cycle, where the position estimates are updated using relative position information obtained from sensors.

\section{A. Position propagation}

The pose of robot $i$ is given by $X_{r_{i}}=\left[x_{r_{i}}, y_{r_{i}}\right]^{T}$, where $x$ and $y$ denote the longitudinal and latitudinal coordinates, respectively. The subscript $r$ is used to distinguish the coordinates of robots from that of landmarks. The discretetime equations approximating the kinematics of robot $i$ are

$$
\begin{aligned}
& x_{r_{i}}(k+1)=x_{r_{i}}(k)+V_{i}(k) \delta t \cos \left(\phi_{i}(k)\right) \\
& y_{r_{i}}(k+1)=y_{r_{i}}(k)+V_{i}(k) \delta t \sin \left(\phi_{i}(k)\right),
\end{aligned}
$$

where $V_{i}(k)$ is the translational velocity at time step $\mathrm{k}$ and $\delta t$ is the sampling time. Let us denote the estimated values for the robot coordinates using a hat $(\hat{\bullet})$, and the corresponding estimation errors by annotating the corresponding quantities with $(\tilde{*})$; for example, $\tilde{\phi}_{i}=\phi_{i}-\hat{\phi}_{i}$. Linearizing (1) along the robot's trajectory, we derive a linear approximation of the robot's position error propagation between time steps

$$
\tilde{X}_{r_{i_{k+1 \mid k}}}=I_{2 \times 2} \tilde{X}_{r_{i_{k \mid k}}}+G_{r_{i}}(k) W_{i}(k),
$$

where $\tilde{X}_{r_{i_{k+1} \mid k}}=\left[\tilde{x}_{r_{i_{k+1 \mid k}}^{T}}^{T} \tilde{y}_{r_{i_{k+1} \mid k}}^{T}\right]^{T}$, and $G_{r_{i}}$ is the error propagation matrix:

$$
G_{r_{i}}(k)=\left[\begin{array}{cc}
\delta t \cos \left(\hat{\phi}_{i}(k)\right) & -V_{m_{i}}(k) \delta t \sin \left(\hat{\phi}_{i}(k)\right) \\
\delta t \sin \left(\hat{\phi}_{i}(k)\right) & V_{m_{i}}(k) \delta t \cos \left(\hat{\phi}_{i}(k)\right)
\end{array}\right],
$$

in which $V_{m_{i}}(k)$ is the measured robot velocity, $\delta t$ is the sampling time and $\hat{\phi}_{i}(k)$ is the estimate of robot's orientation. The term $W_{i}$ is the vector of noise signals, defined as $W_{i}(k)=\left[w_{V_{i}}(k), \tilde{\phi}_{i}(k)\right]^{T}$, where $w_{V_{i}}(k)$ is zero-mean, white Gaussian noise sequence of variance $\sigma_{V_{i}}^{2}$, with $\sigma_{V_{i}}$ being the standard deviation of velocity measurement noise for the $i$ th robot at time step $k$, and $\tilde{\phi}_{i}(k)$ is the error in robot's orientation at time step $k$. From (2), the covariance matrix of system noise for the $i$-th robot is

$$
Q_{r_{i}}(k)=E\left\{G_{r_{i}}(k) W_{i}(k) W_{i}^{T}(k) G_{r_{i}}^{T}(k)\right\} .
$$

In [4] it is shown that the covariance propagation equations of the state error are

$$
P_{k+1 \mid k}=P_{k \mid k}+G Q_{r}(k) G^{T},
$$

where $P_{k \mid k}$ is the covariance matrix of the position errors at time step $k$, calculated based on measurement information collected at step $k$.

\section{B. Position update}

The information obtained from relative measurements between robots is used in the update phase to reduce the position uncertainty of each robot. The relative position measurements obtained when robot $i$ observes robot $j$ are

$$
z_{i j}=C^{T}\left(\phi_{i}\right)\left(X_{i j}-X_{r_{i}}\right)+n_{z_{i j}}
$$

where $X_{i j}$ denotes the position of the robot (or landmark) $j$ observed by robot $i$, and $n_{z_{i j}}$ is the noise affecting this measurement. Matrix $C\left(\phi_{i}\right)$ is a rotation matrix, used to express the relative measurements in the inertial coordinate system. Measurement errors evolve as follows

$$
\tilde{z}_{i j}(k+1)=H_{i j}(k+1) \tilde{X}_{k+1 \mid k}+\Gamma_{i j}(k+1) n_{i j}(k+1),
$$

where $\tilde{X}$ is the combined stack vector of all the estimates for robot and landmark positions, and $\Gamma_{i j}(k)$ is given by

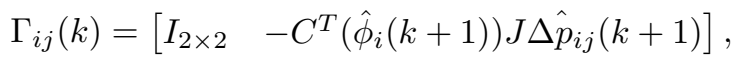

in which

$$
J=\left[\begin{array}{cc}
0 & -1 \\
1 & 0
\end{array}\right], \quad n_{i j}(k)=\left[\begin{array}{c}
n_{z_{i j}}(k) \\
\phi_{i}(k)
\end{array}\right],
$$

and $\Delta \hat{p}_{i j}(k+1)=\hat{X}_{i j_{k+1 \mid k}}-\hat{X}_{r_{i_{k+1 \mid k}}}$, with $\hat{X}_{r_{i}}$ denoting the estimate for position of robot $i$, and $\hat{X}_{i j}$ he position of robot (or landmark) $j$ as estimated by robot $i$.

The matrix $H_{i j}$ is defined in [4] as $H_{i j}(k+1)=$ $C^{T}\left(\hat{\phi}_{i}(k+1)\right) H_{o_{i j}}$, where

$$
H_{o_{i j}}=\left[\begin{array}{llllllll}
0 & \cdots & -I_{2 \times 2} & \cdots & I_{2 \times 2} & 0 & \cdots & 0
\end{array}\right] .
$$

If robot $i$ observes robot $j, H_{o_{i j}}$ is a $2 \times(2 M+2 N)$ matrix with $i$-th entry as $-I_{2 \times 2}$ and $j$-th entry as $I_{2 \times 2}$ and the rest of the entries are zero.

The covariance update equation of the EKF is [4]

$$
P_{k+1 \mid k+1}=P_{k+1 \mid k}-P_{k+1 \mid k} H_{o}^{T} S_{o}^{-1} H_{o} P_{k+1 \mid k},
$$

where $H_{o}$ is a matrix whose block rows are $H_{o_{i}}$, and $H_{o_{i}}$ is a constant matrix with block rows $H_{o_{i j}}$ as given in (3); $S_{o} \triangleq H_{o} P_{k+1 \mid k} H_{o}^{T}+R_{o}(k+1)$, and $R_{o}$ is a block diagonal matrix with elements $R_{o_{i}}$ defined as

$$
\begin{aligned}
R_{o_{i}}=\sigma_{\rho_{i}}^{2} I_{2 M_{i} \times 2 M_{i}} & -D_{i} \operatorname{diag}\left(\frac{\sigma_{\rho_{i}}}{\hat{\rho}_{i j}^{2}}\right) D_{i}^{T} \\
& +\sigma_{\theta_{i}}^{2} D_{i} D_{i}^{T}+\sigma_{\phi_{i}}^{2} D_{i} \mathbf{1}_{M_{i} \times M_{i}} D_{i}^{T},
\end{aligned}
$$

where $D_{i}$ is a block diagonal matrix with diagonal blocks $\Delta \hat{p}_{i j}, M_{i}$ is the number of observations made by robot $i$, and $\sigma_{\rho_{i}}^{2}, \sigma_{\theta_{i}}^{2}, \sigma_{\phi_{i}}^{2}$ are variances of noise in distance measurements, bearing measurements and orientation estimates of robot $i$, respectively. 


\section{Covariance matrix for accurate landmark location}

The system consisting of $M$ robots with kinematics (1) and one landmark becomes observable if the absolute position information of the landmark is available. In this case, the steady-state solution to Riccati recursion becomes [4],

$$
P_{\infty}^{u(0)}=\left[\begin{array}{cc}
P_{r r_{\infty}}^{u} & 0_{2 M \times 2} \\
0_{2 \times 2 M} & 0_{2 \times 2}
\end{array}\right]
$$

The trace of $P_{\infty}^{u(0)}$ serves as a measure of localization accuracy. From (4), note that

$$
\operatorname{trace}\left\{P_{\infty}^{u(0)}\right\}=\operatorname{trace}\left\{P_{r r_{\infty}}^{u}\right\},
$$

and matrix $P_{r r_{\infty}}^{u}$, being symmetric, can be decomposed as

$$
P_{r r_{\infty}}^{u}=Q_{r_{u}}^{\frac{1}{2}} U \operatorname{diag}\left\{\frac{1}{2}+\left(\frac{1}{4}+\frac{1}{\lambda_{i}}\right)^{\frac{1}{2}}\right\} U^{T} Q_{r_{u}}^{\frac{1}{2}},
$$

where $Q_{r_{u}}=\operatorname{diag}\left\{q_{i}\right\}$ with $q_{i}=\max \left\{\delta t^{2} \sigma_{V_{i}}^{2}, \delta t^{2} V_{i}^{2} \sigma_{\phi_{i}}^{2}\right\}$, $U$ is formed by the eigenvectors of $\psi=Q_{r_{u}}^{\frac{1}{2}} I_{r} Q_{r_{u}}^{\frac{1}{2}}$, with $I_{r}$ given by

$$
I_{r}=\left[\begin{array}{ll}
I_{2 M \times 2 M} & 0_{2 M \times 2}
\end{array}\right] H_{o}^{T} R_{u}^{-1} H_{o}\left[\begin{array}{ll}
I_{2 M \times 2 M} & 0_{2 M \times 2}
\end{array}\right]^{T},
$$

and $R_{u}$ is a diagonal matrix

$$
R_{u}=\operatorname{diag}\left\{\left(\sigma_{\rho_{i}}^{2}+M_{i} \sigma_{\phi_{i}}^{2} \rho_{0}^{2}+\sigma_{\theta_{i}}^{2} \rho_{0}^{2}\right) I_{2 M_{i} \times 2 M_{i}}\right\} .
$$

Where $\rho_{0}$ is the maximum distance at which relative measurements can be recorded by the robot. Finally, $\lambda_{i}, i=$ $1, \ldots, 2 M$ are the eigenvalues of $\psi$.

\section{ALGEBRAIC GRAPH THEORETIC CHARACTERIZATION}

To formalize the observation relations we consider a directed graph, which has a node for every robot and landmark in the workspace, and a (directed) edge between a pair of nodes $(a, b)$ whenever the robot associated with one node $a$ observes the object associated with node $b$. The sensing graph is defined as follows:

Definition 1: The sensing graph is a weighted directed graph $\mathcal{X}=\{\mathcal{V}, \mathcal{E}, \mathcal{W}\}$ consisting of

- a set of vertices $\mathcal{V}=\left\{r_{1}, \ldots, r_{M}, L_{1}, \ldots, L_{N}\right\}$, indexed by the $M$ robots and $N$ landmarks,

- a set of edges $\mathcal{E}$, containing ordered pairs of the form $\left(r_{i}, r_{j}\right)$ or $\left(r_{i}, l_{j}\right)$, with $r_{i}, r_{j}, l_{j} \in \mathcal{V}$, representing relative measurements made by the robots, and

- a set of weights, $\mathcal{W}$, consisting of positive constants $\left(\sigma_{\rho_{i}}^{2}+M_{i} \sigma_{\phi_{i}}^{2} \rho_{0}^{2}+\sigma_{\theta_{i}}^{2} \rho_{0}^{2}\right)^{-1}, i \in \mathcal{V}$, each associated with all out-going edges of node $i$.

Our analysis starts with the observation that $H_{o_{i j}}$ in (3) is the Kronecker matrix product of a row of the incidence matrix of $\mathcal{X}, B$, with $I_{2 \times 2}: H_{o_{i j}}=\left(B \otimes I_{2 \times 2}\right)_{i j}$. Then,

$$
H_{o}=B \otimes I_{2 \times 2} \text {. }
$$

Note that according to Definition 1 , matrix $R_{u}^{-1}$ is the diagonal weight matrix of $\mathcal{X}$. The weights associated with each node depend on its out-degree. The weighted Laplacian matrix $L_{w}$ of $\mathcal{X}$ is a positive semidefinite matrix given as

$$
L_{w}=H_{o}^{T} R_{u}^{-1} H_{o} .
$$

The smallest eigenvalue of $L_{w}$ is zero and the rest are always positive [14].

Note that $I_{r}$ in (6) is obtained by multiplication of $L_{w}$ by $I_{y}=\left[\begin{array}{ll}I_{2 M \times 2 M} & 0_{2 M \times 2}\end{array}\right]$ from both sides. Such an operation results in the removal of the last row (and column) from $L_{w}$. This is the row (and column) corresponding to the known landmark. Due to matrix tree theorem $I_{r}$ becomes positive definite [14], denoted $L_{w}[M+N]$ (following the notation of [14]). Using (5),

$$
\begin{aligned}
& \operatorname{trace}\left\{P_{r r_{\infty}}^{u(0)}\right\}= \\
& \operatorname{trace}\left\{Q_{r_{u}}^{\frac{1}{2}} U \operatorname{diag}\left\{\frac{1}{2}+\left(\frac{1}{4}+\frac{1}{\lambda_{i}}\right)^{\frac{1}{2}}\right\} U^{T} Q_{r_{u}}^{\frac{1}{2}}\right\} .
\end{aligned}
$$

Using the properties of the trace [15],

$$
\begin{aligned}
& \operatorname{trace}\left\{P_{r r_{\infty}}^{u(0)}\right\} \\
& =\operatorname{trace}\left\{U^{T} Q_{r_{u}} U \operatorname{diag}\left\{\frac{1}{2}+\left(\frac{1}{4}+\frac{1}{\lambda_{i}}\right)^{\frac{1}{2}}\right\}\right\} .
\end{aligned}
$$

Matrix $U^{T} Q_{r_{u}} U$ is positive definite since $Q_{r u}$ is a diagonal matrix, and the diagonal elements are strictly positive.

Let $\alpha_{i}$ denote the $i$ th diagonal element of $U^{T} Q_{r_{u}} U$ and $\mu_{i}$ the $i$ th diagonal element of $\operatorname{diag}\left\{\frac{1}{2}+\left(\frac{1}{4}+\frac{1}{\lambda_{i}}\right)^{\frac{1}{2}}\right\}$. Then, from (8) it follows that

$$
\operatorname{trace}\left\{P_{r r_{\infty}}^{u}\right\}=\sum_{i=1}^{2 M} \alpha_{i} \mu_{i} \Rightarrow \frac{\partial \operatorname{trace}\left\{P_{r r_{\infty}}^{u}\right\}}{\partial \mu_{i}}=\alpha_{i} \geq 0 .
$$

Thus, the trace of $P_{r r_{\infty}}^{u}$ increases monotonically with each $\mu_{i}$. Since

$$
\mu_{i}=\frac{1}{2}+\left(\frac{1}{4}+\frac{1}{\lambda_{i}}\right)^{\frac{1}{2}}
$$

where $\lambda_{i}$ are the eigenvalues of $\psi$, the larger $\lambda_{i}$ are, the more accurate the position estimates will likely be. Note now that $\mu_{i} \leq 1+\frac{1}{\sqrt{\lambda_{i}}}$, so that

$$
\operatorname{trace}\left\{P_{r r_{\infty}}^{u}\right\} \leq \operatorname{trace}\left\{U^{T} Q_{r_{u}} U \operatorname{diag}\left\{1+\frac{1}{\sqrt{\lambda_{i}}}\right\}\right\} \text {. }
$$

To see how we can manipulate $\lambda_{i}$, note that

$$
\operatorname{trace}\{\psi\}=\operatorname{trace}\left\{Q_{r_{u}}^{\frac{1}{2}} I_{r} Q_{r_{u}}^{\frac{1}{2}}\right\}=\operatorname{trace}\left(Q_{r_{u}} I_{r}\right),
$$

and if $q_{i}$ is the $i$ th diagonal element of $Q_{r_{u}}$, and $i_{i i}$ is the $i$ th diagonal element of matrix $I_{r}$, then

$$
\operatorname{trace}\{\psi\}=\sum_{i=1}^{2 M} q_{i} i_{i i} \Rightarrow \frac{\partial \operatorname{trace}\left\{Q_{r_{u}} I_{r}\right\}}{\partial i_{i i}}=q_{i}>0 .
$$

Hence, to minimize $P_{r r_{\infty}}^{u}$, it suffices that the eigenvalues of $I_{r}$ are increased. The eigenvalues of $I_{r}$ (and therefore $\psi$ ) are positive.

Matrix $I_{r}$ is obtained from $L_{w}$ after removing the two rows and columns associated with the landmark. The eigenvalues of $I_{r}$, therefore, interlace those of $L_{w}$ [15], since $I_{r}$ is 
a principal submatrix of $L_{w}$. This suggests that if the eigenvalues of $L_{w}$ increase, those of $I_{r}$ have to increase too, indicating a way to regulate the trace of $P_{r r_{\infty}}^{u}$ : enforce sensing graph topologies that increase the spectrum of $L_{w}$.

In the case of an unweighted graph, the eigenvalues of the Laplacian always increase with the addition of an edge [14]. What we show in section $\mathrm{V}$ is that the same happens for the case of weighted graphs. This is a fairly well established result in the graph theory community; what section $\mathrm{V}$ does is to offer the proofs that appear to be missing from [16].

Combined with weighted graph interlacing, (9) is in complete agreement with [13]: there, the genetic algorithm suggested a complete graph as the best sensing topology. However, if the complete graph topology is infeasible, or too expensive to implement, (9) provides another means to achieve the required accuracy: if one is to add a single edge to the sensing graph, (9) points to the robot that should do the observation. What the particular robot should observe, depends on the topology of the sensing graph, as expressed by the spectrum of the Laplacian.

\section{HOW TO CONTROL THE COVARIANCE TRACE}

\section{A. Interlacing for Weighted Graphs}

Interlacing is a relationship between the eigenvalues of a matrix and those of its submatrix. Suppose $A$ is a real, symmetric $m \times m$ matrix and $B$ is a real, symmetric $n \times n$ submatrix of $A$. Then the eigenvalues of $B$ interlace the eigenvalues of $A$. Let $\left\{\lambda_{1}, \ldots, \lambda_{n}\right\}$ denote the eigenvalues of $A$, and $\left\{\theta_{1}, \ldots, \theta_{n}\right\}$ be the eigenvalues of $B$. Then, $\lambda_{n-m+i}(A) \leq \theta_{i}(B) \leq \lambda_{i}(A)[14]$.

In this section, we offer proofs for some of the interlacing results on the Laplacian spectrum of weighted graphs that appear in [16]. This section does not introduce new theory; besides giving the proofs, it provides insight into how these known results apply to the particular case of cooperative localization using relative measurements. We start with proving that the smallest positive eigenvalue of the new weighted Laplacian matrix obtained by adding an edge to the original one is always greater than the smallest positive eigenvalue of the original graph.

Theorem 2: Let $\mathcal{X}$ be a graph with $\mathcal{V}$ vertices and $\mathcal{E}$ edges with weights $w_{i j}$ on each edge, and let $Q(\mathcal{X})$ be the weighted Laplacian matrix of $\mathcal{X}$. Let graph $\mathcal{Y}$ be obtained by adding an edge to $\mathcal{X}$, joining two distinct vertices of $\mathcal{X}$. Then, $\lambda_{2}(\mathcal{X}) \leq$ $\lambda_{2}(\mathcal{Y})$

Proof: Assume that $\mathcal{Y}$ is obtained by joining vertices $r$ and $s$ of $\mathcal{X}$,and let $Q(\mathcal{Y})$ denote the weighted Laplacian of $\mathcal{Y}$. For any eigenvector $z$ orthogonal to $\mathbf{1}$ (the vector of ones), we have similarly to [14], [17],

$$
\begin{aligned}
\lambda_{2}(\mathcal{Y})= & \min _{z^{T} \mathbf{1}=0} \frac{z^{T} Q(\mathcal{Y}) z}{z^{T} z} \\
= & \min _{z^{T} \mathbf{1}=0} \frac{w_{r s}\left(z_{r}-z_{s}\right)^{2}+\sum_{u v \in \mathcal{E}(\mathcal{X})} w_{u v}\left(z_{u}-z_{v}\right)^{2}}{z^{T} z} \\
= & \min _{z^{T} \mathbf{1}=0} \frac{w_{r s}\left(z_{r}-z_{s}\right)^{2}}{z^{T} z} \\
& +\min _{z^{T} \mathbf{1}=0} \frac{\sum_{u v \in \mathcal{E}(\mathcal{X})} w_{u v}\left(z_{u}-z_{v}\right)^{2}}{z^{T} z} \\
= & \min _{z^{T} \mathbf{1}=0} \frac{w_{r s}\left(z_{r}-z_{s}\right)^{2}}{z^{T} z}+\lambda_{2}(\mathcal{X})
\end{aligned}
$$

Hence, $\lambda_{2}(\mathcal{Y}) \geq \lambda_{2}(\mathcal{X})$.

Thus, the smallest eigenvalue always increases with addition of an edge to the original graph. Increasing $\lambda_{2}$ only affects one of the terms in the sum that produces trace $\left\{P_{r r_{\infty}}^{u}\right\}$. In the next theorem we prove that all the eigenvalues of the new weighted Laplacian increase with addition of an edge. We make use of the following Lemma from [15]:

Lemma 3 ([15]): Let $A, B$ be Hermitian matrices. For a positive definite matrix $B$, the following is always true $\lambda_{k}(A) \leq \lambda_{k}(A+B)$.

The next theorem extends the interlacing property to weighted Laplacians.

Theorem 4: Let $\mathcal{X}$ be a weighted sensing graph with $n$ vertices and $\mathcal{Y}$ be obtained from $\mathcal{X}$ by adding an edge joining two distinct vertices of $\mathcal{X}$. If the weights on the edges of $\mathcal{Y}$ that are also edges in $\mathcal{X}$ do not decrease, then, $\lambda_{i}(\mathcal{X}) \leq$ $\lambda_{i}(\mathcal{Y})$, for all $i=1, \ldots, n$.

Proof: Let $\mathcal{Y}$ be the graph obtained by adding an edge $e$ joining vertices $i$ and $j$ of graph $\mathcal{X}$. The degree of vertex $i$ then increases from $M_{i}$ to $M_{i}+1$. Expressing the weighted graphs $\mathcal{X}$ and $\mathcal{Y}$ as

$$
\mathcal{X}=\{\mathcal{V}, \mathcal{E}, \mathcal{W}\}, \quad \mathcal{Y}=\left\{\mathcal{V}, \mathcal{E} \cup\{e\}, \mathcal{W} \cup\left\{w_{e}\right\}\right\}
$$

Let $W(\mathcal{X})=\operatorname{diag}\left\{a_{k}\right\}, a_{k} \in \mathcal{W}$ denote the diagonal weight matrix of $\mathcal{X}$ and define the augmented matrix

$$
W(\mathcal{X})^{+} \triangleq\left[\begin{array}{ll}
W(\mathcal{X}) & 0 \\
0_{1 \times|\mathcal{E}|} & 0
\end{array}\right]=\left[\begin{array}{ccc|c}
{\left[\begin{array}{ccc}
\ddots & & \\
& a_{k} & \\
& & \ddots
\end{array}\right]} & 0 \\
\hline 0 & \cdots & 0 & 0
\end{array}\right] .
$$

Rearrange the weights in the weight matrix of $\mathcal{Y}$, so that the new edge, $e$, weight appears last:

$$
W(\mathcal{Y})=\operatorname{diag}\left\{\ldots, b_{k}, \ldots, e_{w}\right\} .
$$

If $H_{o}(\mathcal{Y})=B(\mathcal{Y}) \otimes I_{2}$, where $B(\mathcal{Y})$ is the incidence matrix of $\mathcal{Y}$, then note that one can write the weighted Laplacian matrix of $\mathcal{Y}$ as

$$
Q(\mathcal{Y})=Q(\mathcal{X})+H_{o}(\mathcal{Y})^{T}\left[W(\mathcal{Y})-W(\mathcal{X})^{+}\right] H_{o}(\mathcal{Y})
$$

and that the second term on the right hand side is a weighted Laplacian too, provided that $b_{k}-a_{k} \geq 0$. Being a (weighted) Laplacian, $H_{o}(\mathcal{Y})^{T}\left[W(\mathcal{Y})-W(\mathcal{X})^{+}\right] H_{o}(\mathcal{Y})$ is 
positive semidefinite. Thus, by Lemma 3, the eigenvalues of $Q(\mathcal{Y})$ interlace those of $Q(\mathcal{X})$.

Theorem 4 is interesting on its own, but of limited use in the particular application where the weight matrix is expressed as $R_{u}^{-1}$, and its elements are given by the inverses of the diagonal elements of (7). For the addition of an edge, reduces the diagonal elements in $R_{u}^{-1}$ that are associated with edges in $\mathcal{X}$ originating from $i$, and the condition $b_{k} \geq$ $a_{k}$ no longer holds.

Thus, in the case of sensing graphs, the addition of an edge does not necessarily increase all the eigenvalues of $I_{r}$, $\lambda_{i}$. However, it turns out that the introduction of the weight of the new edge is sufficient to increase the trace of the weighted Laplacian:

Theorem 5: Let $\mathcal{X}$ be a weighted sensing graph with $n$ vertices and $\mathcal{Y}$ be obtained from $\mathcal{X}$ by adding an edge joining two distinct vertices of $\mathcal{X}$. Then, $\operatorname{trace}\{Q(\mathcal{Y})\} \geq$ $\operatorname{trace}\{Q(\mathcal{X})\}$.

Proof: Suppose a new edge $e$ is added to the graph $\mathrm{X}$ when robot $i$ observes robot $j$. The dimension of the diagonal block corresponding to the $i$-th robot in the diagonal weight matrix becomes $2\left(M_{i}+1\right) \times 2\left(M_{i}+1\right)$, where $M_{i}$ denotes the number of measurements made by the $i$-th robot before the addition of this new edge. Define the reduced diagonal weight matrix of $\mathcal{Y}, W(\mathcal{Y}[e])$, as the one obtained from $W(\mathcal{Y})$ ) when the row and column corresponding to the newly added edge $e$ is removed. The new weighted Laplacian can be written as

$Q(\mathcal{Y})=Q(\mathcal{X})+H_{o}^{T}(\mathcal{X})[W(\mathcal{Y}[e])-W(\mathcal{X})] H_{o}(\mathcal{X})+b P P^{T}$

where $P$ is the new (block) column (associated to edge $e$ ) added to the matrix $H_{o}(\mathcal{X})$ to obtain $H_{o}(\mathcal{Y})$. Then,

$$
\begin{aligned}
& \operatorname{trace}\{Q(\mathcal{Y})\}=\operatorname{trace}\{Q(\mathcal{X})\} \\
& \quad+\operatorname{trace}\left\{H_{o}^{T}(\mathcal{X})[W(\mathcal{Y}[e])-W(\mathcal{X})] H_{o}(\mathcal{X})\right\} \\
&+\operatorname{trace}\left\{b P P^{T}\right\} .
\end{aligned}
$$

From the above, we express the difference between the traces of $Q(\mathcal{Y})$ and $Q(\mathcal{X})$, as the trace of some another diagonal matrix, $T$

$$
\begin{aligned}
& \operatorname{trace}\{T\} \\
& =\operatorname{trace}\left\{H_{o}^{T}(\mathcal{X})[W(\mathcal{Y}[e])-W(\mathcal{X})] H_{o}(\mathcal{X})+b P P^{T}\right\}
\end{aligned}
$$

the elements of which are shown by direct calculation to satisfy

$$
\operatorname{trace}(T) \propto\left(\sigma_{\rho_{i}}^{2}+\left(M_{i}-1\right) \sigma_{\phi_{i}}^{2} \rho_{o}^{2}+\sigma_{\theta_{i}}^{2} \rho_{o}^{2}\right) .
$$

Since $M_{i} \geq 1$, and all the other terms in the right hand side are positive, it follows that $\operatorname{trace}\{Q(\mathcal{Y})\} \geq \operatorname{trace}\{Q(\mathcal{X})\}$.

\section{B. Discussion}

Together, Theorems 2 and 5 give us a handle to manipulate the trace of $P_{r r_{\infty}}^{u}$, through $I_{r}$, viewing the latter as the principal submatrix of the weighted Laplacian of the sensing graph. Inequalities relating the harmonic mean, the minimum element, and the arithmetic mean of a set of numbers, applied to the eigenvalues of $I_{r}$ yield

$$
\min \left\{\lambda_{1}, \ldots, \lambda_{n-1}\right\} \leq \frac{1}{\frac{1}{n-1} \sum_{i=2}^{n-1} \frac{1}{\lambda_{i}}} \leq \frac{1}{n-1} \sum_{i=2}^{n-1} \lambda_{i}
$$

In view of $I_{r}$ being the principal submatrix of a weighted Laplacian $L_{w}$, it follows

$$
\frac{(n-1)^{2}}{\sqrt{\operatorname{trace}\left\{L_{w}\right\}}} \leq \sum_{i=1}^{n-1} \frac{1}{\sqrt{\lambda_{i}}} \leq \frac{n-1}{\sqrt{\lambda_{2}\left(L_{w}\right)}} .
$$

Thus, increasing the smallest nonzero eigenvalue of $L_{w}$ along with its trace, pushes the trace of the error covariance matrix for the system to lower values. The increase in $\lambda_{2}\left(L_{w}\right)$ restricts the covariance trace from above, while the increase in the trace of $L_{w}$ allows it to decrease without saturating at a lower bound.

\section{EXAMPLES}

Figure 1 shows several ways to add an edge to a sensor graph, in other words, allow a robot to make an additional relative position measurement. The directed graph shown in

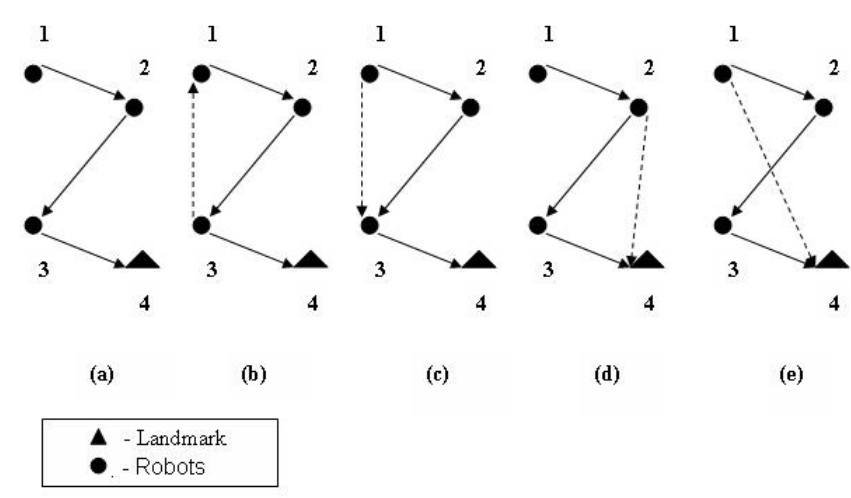

Fig. 1. Sensing graphs of three robots and one landmark with different topologies. Solid arrows indicate original observations; the dotted arrow show the additional measurement.

1(a), (denoted henceforth $\mathcal{G}_{a}$ ) is thought of as the original graph consisting of four nodes, where nodes 1,2 and 3 represent robots and node 4 is a known landmark. Robot 1 observes robot 2 , robot 2 observes robot 3 , and robot 3 makes relative position measurements with respect to the landmark. The incidence matrix $B$, (from which $H_{o}$ is derived) for this graph is given by:

$$
B=\left[\begin{array}{cccc}
-1 & 1 & 0 & 0 \\
0 & -1 & 1 & 0 \\
0 & 0 & -1 & 1
\end{array}\right]
$$

The weight matrix $R_{u}$ is a diagonal matrix of size $2\left(\sum_{i=1}^{3} M_{i}\right) \times 2\left(\sum_{i=1}^{3} M_{i}\right)$ with the diagonal block element $i$ being of size $2 M_{i} \times 2 M_{i}$ : $\operatorname{diag}\left\{a=\left(\sigma_{\rho}^{2}+M_{i} \sigma_{\phi}^{2} \rho^{2}+\sigma_{\theta}^{2} \rho^{2}\right)\right\}$. Assuming all robots being homogeneous, for $\mathcal{G}_{a}$ the diagonal elements in the weight matrix $R_{u}$ are the same. Hence, $R_{u}=\operatorname{diag}\{a, a, a, a, a, a\}$. 
Taking $\sigma_{\rho}=0.05, \sigma_{\phi}=2^{\circ}, \sigma_{\theta}=2^{\circ}, \rho_{o}=6 \mathrm{~m}$, yields $a=0.0902$ for $M_{i}=1$. The weighted Laplacian matrix $L_{w}$ for $\mathcal{G}_{a}$ is $L_{w}=H_{o}^{T} R_{u}^{-1} H_{o}$, and the corresponding principal submatrix $L_{w}[x]$ is obtained by deleting the last two rows and columns of $L_{w}$. For $L_{w}[x]\left(\mathcal{G}_{a}\right)$ we have

$$
\begin{aligned}
& \sigma_{a}=\{2.1958,2.1958,17.239,17.239,35.9976,35.9976\} \\
& \operatorname{trace}\left\{L_{w}[x]\right\}=110.8648, \\
& \sum_{i=1}^{6} \frac{1}{\sqrt{\lambda_{i}}}=2.164 .
\end{aligned}
$$

Figures 1(b)-(e) show four possibilities for the addition of an edge. Weights change according to the topology of the resulting graph. Table I compares the eigenvalues, the trace, and the quantity $\sum \frac{1}{\sqrt{\lambda_{i}}}$ obtained for $L_{w}[x]$ for each of the

\begin{tabular}{|c|c|c|c|c|}
\hline case & $\lambda_{i}$ & $\operatorname{tr}(L[x])$ & $\sum \frac{1}{\sqrt{\lambda_{i}}}$ & $\operatorname{tr}\left(P_{r r_{\infty}}^{u}\right)$ \\
\hline (a) & $\begin{array}{l}2.1958,17.2390,35.9976 \\
2.1958,17.2390,35.9976\end{array}$ & 110.8648 & 2.164 & 3.0398 \\
\hline (b) & $\begin{array}{l}2.0788,29.0788,35.5607 \\
2.0788,29.0788,35.5607\end{array}$ & 133.4346 & 2.0934 & 3.0228 \\
\hline (c) & $\begin{array}{l}2.8394,23.4351,36.8155 \\
2.8394,23.4351,36.8155\end{array}$ & 126.1758 & 1.8936 & 2.9226 \\
\hline (d) & $\begin{array}{l}4.2196,16.7477,34.6635 \\
4.2196,16.7477,34.6635\end{array}$ & 111.2616 & 1.802 & 2.8378 \\
\hline (e) & $\begin{array}{l}5.3858,17.2583,32.9867 \\
5.3858,17.2583,32.9867\end{array}$ & 111.2616 & 1.6914 & 2.7798 \\
\hline
\end{tabular}
cases (b) through (e), as well as the trace of the steady state covariance matrix.

TABLE I

COMPARING EIGENVALUES AND TRACE

Table I shows that that trace of principal submatrix of graphs obtained by edge addition $\left(\mathcal{G}_{b}\right.$ through $\mathcal{G}_{e}$ ) is always higher than that of $\mathcal{G}_{a}$. Similarly, the values of $\sum \frac{1}{\sqrt{\lambda_{i}}}$ and trace $\left\{P_{r r_{\infty}}^{u}\right\}$ are smaller for all new graphs compared to $\mathcal{G}_{a}$. Note that the graph with the highest smallest eigenvalue, $\mathcal{G}_{e}$, (see Figure 1(e)) has the lowest value of $\sum \frac{1}{\sqrt{\lambda_{i}}}$, and therefore the smallest trace for $P_{r r_{\infty}}^{u}$. Interestingly, the poorest choice in terms of the smallest eigenvalue for $L_{w}[x]$ is that of Figure 1(b), however, even in this case the trace of the steady state covariance matrix is still better than that of the original configuration. However, if one has to choose to connect two nodes with one observation, it is clear that the new edge in $\mathcal{G}_{e}$ is the optimal. Intuitively, it can be justified by the fact that now robot 1 has direct access to accurate position information, by measuring its own with respect to the known landmark. Our main point is that investigation of graph topologies with respect to their Laplacian spectra might give us additional insight on how to construct efficient (smaller) sensing graphs that meet specific accuracy specifications.

\section{CONCLUSION}

This paper provides insight to the way in which network connectivity affects the cooperative localization accuracy. We have demonstrated that the topology of the sensing graph, as expressed by the spectrum of the associated weighted Laplacian, determines the accuracy of localization, if the latter is quantified by the trace of the covariance matrix associated with robot position estimates. Although "denser" graphs generally result in increased localization accuracy, in agreement with [13], when it comes to choosing how to add additional observations, some links are better than others. The analysis provided in this paper facilitates this choice by indicating efficient ways of increasing accuracy per (new) observation.

\section{AKNOWLEDGMENT}

The work of the second author is supported in part by the NSF CISE Career award \# 0447898, and in part by DoE URPR grant DE-FG52-04NA25590.

\section{REFERENCES}

[1] J. Borenstein and L. Feng, "Measurement and correction of systematic odometry errors in mobile robots," IEEE Transactions on Robotics and Automation, vol. 12, no. 6, pp. 869-880, 1996.

[2] F. Giuffrida, C. Massucco, P. Morasso, G. Vercelli, and R. Zaccaria, "Multi-level navigation using active localization system," in IEEE International Conference on Intelligent Robotics and Systems, vol. 1, 1995, pp. 413-418.

[3] C. Lin and R. Tummala, "Mobile robot navigation using artificial landmarks," Journal of Robotic Systems, vol. 14, no. 2, pp. 93-106, 1991.

[4] A. Mourikis and S. Roumeliotis, "Performance bounds for cooperative simultaneous localization and mapping," in Robotics: Science and Systems, 2005, pp. 73-80.

[5] S. Roumeliotis and G. Bekey, "Distributed multi-robot localization," Distributed Autonomous Robotic Systems, vol. 4, pp. 179-188, 2000.

[6] S. Thrun, D. Fox, W. Burgard, and F. Dellaert, "Robust monte carlo localization for mobile robots," Artificial Intelligence, vol. 128, no. 1-2, pp. 99-141, 2000.

[7] W. Burgard, D. Fox, M. Moors, R. Simmons, and S. Thrun, "Collaborative multi-robot exploration," in IEEE International Conference on Robotics and Automation, vol. 1, 2000, pp. 476-481.

[8] D. Fox, "Markov localization: A probabilistic framework for mobile robot localization and navigation," Ph.D Dissertation, University of Bonn, 1998.

[9] W. Burgard, D. Fox, D. Henning, and T. Schmidt, "Estimating the absolute position of a mobile robot using position probability grids," in National Conference on Artificial Intelligence, vol. 2, 1996, pp. 896-901.

[10] S. Thrun, "A probabilistic online mapping algorithm for teams of mobile robots," International Journal of Robotics Research, vol. 20, pp. 335-363, 2001 .

[11] W. Burgard, D. Fox, H. Jans, C. Matenar, and S. Thrun, "Sonarbased mapping of large-scale mobile robot environments using EM," in International Conference on Machine Learning, 1999, pp. 67-76.

[12] R. Kurazume, S. Nagata, and S. Hirose, "Cooperative positioning with multiple robots," in IEEE International Conference on Robotics and Automation, vol. 2, 1994, pp. 1250-1257.

[13] Y. Hidaka, A. Mourikis, and S. Roumeliotis, "Optimal sensing strategies for mobile robot formations," in Robotics: Science and Systems, 2005, pp. 281-288.

[14] C. Godsil and G. Royle, Algebraic Graph Theory. Springer, 2001.

[15] R. Horn and C. Johnson, Matrix Analysis. Cambridge, 1999.

[16] B. Mohar, "Laplacian spectrum of graphs," Graph Theory, Combinatorics and Applications, vol. 2, pp. 871-898, 1991.

[17] S. Guattery and G. Miller, "Graph embeddings and laplacian eigenvalues," Siam J. Matrix Anal. and Applications, vol. 21, no. 3, pp. 703-723, 2000. 